\section{TOOTHPASTE HARDENS YOUR TEETH WHILE YOU SLEEP}

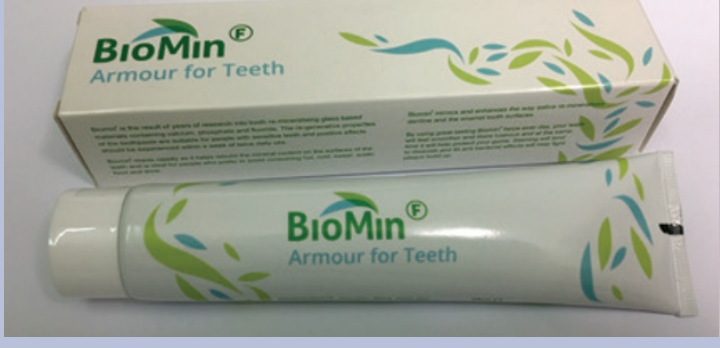

A new toothpaste ingredient which puts back the lost minerals from tooth enamel, helps prevent decay and treats sensitivity while you sleep is now available.

Tooth decay and sensitivity is estimated to affect 13.5 million people in the UK alone. Toothpastes containing BioMinF are able to slowly release calcium, phosphate and fluoride ions over an 8-12 hour timeframe to form fluorapatite mineral to rebuild, strengthen and protect tooth structure. The slow release of fluoride has been identified to be particularly beneficial in prevention of tooth decay.

Using remineralising toothpaste makes teeth far more resistant to attack from acidic soft drinks like fruit juices and sodas. It is also much more effective than conventional toothpastes where the active ingredients, such as soluble fluoride, are washed away and become ineffective less than two hours after brushing. The technology behind BioMin can also be incorporated in other professionally applied dental products such as cleaning and polishing pastes, varnishes and remineralising filling materials.

The team behind BioMin are in the process of establishing licensing agreements with toothpaste and dental materials manufacturers around the world. A fluoride-free version of BioMin is also being developed for those who do not want or need fluoride toothpaste.

BioMinF toothpaste is available priced $£ 4.99$ for a $75 \mathrm{ml}$ tube at dental practices who can obtain the product via dental wholesalers (call Trycare on 01274881044 or Serve-ice on 01483 751789) or find out more at www.biomin.co.uk.

\section{EXPERT BUSINESS SOLUTIONS}

Everyone is welcome to stand B08 to meet the friendly and knowledgeable team of experts ready to offer advice on all aspects of surgery and decontamination equipment, lasers, digital technology, servicing, business support and much more.

Henry Schein's Business Solutions will be introducing Practice Analysis, a proven process for analysing practice performance and identifying solutions to help practice owners meet their professional goals.

By using specific data from the practice management software, Practice Analysis is able to show practice

\title{
THE BEST DENTAL JOBS SITE IS ABOUT TO GET BETTER
}

The new and improved BDJ Jobs site is about to launch - watch this space!

Whether you're looking for a new dentist, specialist, locum or dental care professional (DCP) position, with the brand new BDJ Jobs site, it will be easier than ever to find your perfect job.

The new www.bdjjobs.com will have improved search functionality allowing you to filter by specific criteria, including location, job, organisation and practice type. You will be able to create a shortlist and set up job alerts to ensure you get targeted vacancies delivered directly to your inbox.

With the new site, you can be more visible to prospective employers by uploading your $\mathrm{CV}$, even if there is no immediate vacancy. The new $B D J$ Jobs website will also have a sleeker interface and mobile-friendly design, so you can apply for jobs directly from your phone and receive new job alerts wherever you are.

The new site will boast a library of essential dental careers advice and accessible workplace research to help you plan your next career move.

If you are a recruiter, improved searchability will make your vacancy easier to find and your job will be emailed directly to candidates specifically interested in your type of vacancy. It will be seen by over 25,000 unique web users every month (Webtrends, March 2016).

To find out more about BDJ Jobs and other fantastic offerings from the $B D J$ portfolio, visit stand B35 at this year's British Dental Conference and Exhibition.

\section{CLAIMS AGAINST DENTAL PROFESSIONALS HAVE QUADRUPLED}

The number of claims against dental professionals exceeding $£ 100,000$ has quadrupled, according to the Dental Defence Union (DDU), with 11 claims for more than $£ 100,000$ settled on behalf of members in 2015, as opposed to just two in 2006.

Over the last ten years, the DDU paid out nearly £5 million to compensate patients and pay legal costs in claims that exceeded $£ 100,000$. The highest compensation amount was $£ 120,000$. Other cases exceeded $£ 200,000$ when claimant's legal fees were added.

John Makin, Head of the DDU, said that these payments are no reflection on clinical standards, but a result of rising expectations and availability of 'no win no fee' arrangements.

Over the last three years the DDU has paid more in claimant's legal costs than in compensation and the increases in the size and number of claims is leading to indemnity becoming more expensive for dental professionals.

While the current climate is challenging for dental professionals, who are more likely than ever to face a claim, the DDU has made no compensation payment in over 50\% of cases in the last 10 years.

www.theddu.com owners how their business is performing against where the practice owner believes they should be, and offer solutions that that can make a tangible impact on business revenue and practice efficiency.

Analysing practice performance in areas key to revenue generation including treatment analysis and recall effectiveness shows exactly what is happening in any individual practice. Working closely with the practice principal Henry Schein's commitment is to offer the practice tailor-made solutions where any shortfalls may occur and establish the priorities that will help the practice realise their full potential.

The standard cost of a Practice Analysis is $£ 249.00$ + VAT. Bookings made up to and including the BDA Conference will be at the introductory price of $£ 199.00+$ VAT.

Visit hsbusinesssolutions.co.uk. 